\title{
Correlation Analysis between the Level of Coagulation Function and Osteonecrosis of Femoral Head
}

\author{
H. WANG AND L. YAN*
}

Clinical School of Yangzhou University, Yangzhou 225001, Jiangsu, 'Department of Orthopedics, Sihong People's Hospital, Sihong 223900, Jiangsu Province, China

Wang et al.: Correlation between the Coagulation Function and Osteonecrosis of Femoral Head

\begin{abstract}
To explore the correlation analysis between the level of coagulation function and osteonecrosis of femoral head is the main objective of the study. Osteonecrosis of femoral head is the end point of the disease process due to inadequate blood flow and osteonecrosis of bone tissue and later in the disease, femoral head collapse, arthritis or even hip replacement surgery may be required. Therefore, it is called avascular necrosis of femoral head and is a common and intractable disease in orthopedics clinic. According to statistics, there are about 7.5 10 million patients with osteonecrosis of femoral head in our country and the number of new cases is up to 300000 every year. It is seen mostly in people aged $30-50 \mathrm{y}$ and about half involve bilateral femoral heads. Its pathogenesis is influenced by many factors and complex mechanisms, and osteonecrosis of femoral head is most commonly caused by trauma, corticosteroid and alcohol use, but is also associated with blood metabolic disorders and coagulation disorders. To evaluate the relationship between prothrombin time, activated partial thromboplastin time, thrombin time and fibrinogen levels in patients with osteonecrosis of femoral head. Prothrombin time, activated partial thromboplastin time and fibrinogen levels were independent risk factors for the development of osteonecrosis of femoral head and all of them were risk factors; that is, the lower the prothrombin time and activated partial thromboplastin time levels, the higher the fibrinogen level and the higher the risk of osteonecrosis of femoral head occurrence.
\end{abstract}

Key words: Coagulation function, femoral head, necrosis, logistic regression analysis

Osteonecrosis of Femoral Head (ONFH) is the end point of the disease process due to inadequate blood flow and osteonecrosis of bone tissue and later in the disease, femoral head collapse, arthritis or even hip replacement surgery may be required. Therefore, it is called Avascular Necrosis of Femoral Head (AVNFH) and is a common and intractable disease in orthopedics clinic $^{[1,2]}$. According to statistics, there are about 7.5 10 million patients with ONFH in our country and the number of new cases is up to 300000 every year. It is seen mostly in people aged 30-50 y and about half involve bilateral femoral heads. Its pathogenesis is influenced by many factors and complex mechanisms, and ONFH is most commonly caused by trauma, corticosteroid and alcohol use, but is also associated with blood metabolic disorders and coagulation disorders ${ }^{[3,4]}$. Often it is divided into traumatic and nontraumatic factors; the former is mainly caused by hip trauma, which results from acute mechanical disruption of the blood supply to the femoral head ${ }^{[5]}$; whereas for the latter non-traumatic ONFH, many hypotheses have been proposed that hormonal and other etiologies lead to hypertrophy of marrow adipocytes, an increase in bone marrow pressure and ultimately intravascular coagulation and thromboembolism within the microcirculation, resulting in osteonecrosis ${ }^{[6]}$. Previous studies have shown that in patients with non-traumatic $\mathrm{ONFH}$, the occurrence and development process are closely related to the metabolism of blood lipids, blood diseases, blood cell levels, coagulation disorders, etc. ${ }^{[7-}$ ${ }^{10]}$ while apart from these common causes of ONFH, there are some unexplained studies on the relationship between idiopathic ONFH and coagulation function have rarely been reported, so the coagulation indices in this subset of patients with idiopathic ONFH were studied with intergroup contrast and are reported below.

*Address for correspondence

E-mail: xiaohaozi0527@163.com 


\section{MATERIALS AND METHODS}

\section{General data:}

Through the hospital electronic medical record system, 224 patients with ONFH who met the diagnostic, inclusion and exclusion criteria of this study were selected as the experimental group from June 2019 to June 2021 in the Department of Osteoarthritis, the Northern Jiangsu People's Hospital. A total of 243 consecutive hospitalized femoral neck fracture patients during the same period were selected as the control group. In the experimental group, there were 94 males and 130 females, aged $28-88 \mathrm{y}$, with a mean age of $65.31 \pm 10.76$ y. In the control group, there were 64 males and 179 females, aged 51-98 y, with a mean age of $75.39 \pm 9.63$ y. The inclusion criteria (met simultaneously) includes the diagnostic criteria were based on the diagnostic criteria of ONFH proposed by Professor Zhao Dewei ${ }^{[2]}$; fulfilled the above diagnostic criteria and age $\geq 18 \mathrm{y}$. Exclusion criteria $\geq 18$ y. Exclusion criteria: patients patients with chronic diseases (hematological diseases, chronic kidney disease, chronic hepatitis, cirrhosis, thrombotic disease, and cancer); history of alcohol abuse (average consumption of pure alcohol $\geq 400 \mathrm{~g}$ per week during drinking period); history of steroid use; history of hip infection, tuberculosis, congenital hip dysplasia; history of anticoagulant use such as aspirin, warfarin, clopidogrel and low molecular weight heparin calcium within $1 \mathrm{w}$ prior to blood draw; patients with femoral neck fractures whose disease duration was greater than $3 \mathrm{w}$; those had been operated for this ONFH or femoral neck fracture prior to testing; those whose basic information and coagulation indicators were missing; those patients had both ONFH and femoral neck fracture; there was a clear history of trauma to the ipsilateral hip in the patient with ONFH.

\section{Methods:}

The patients who were admitted to the Department of Osteoarthritis from June 2019 to June 2021 with "Aseptic necrosis of the femoral head" as the admission diagnosis were retrieved through the Hospital Information System (HIS) system of the Northern Jiangsu People's Hospital and the basic information such as age, gender, cause of disease and the tested coagulation index were collected from the medical records of all study subjects: Activated Partial Thromboplastin Time (APTT), Prothrombin Time (PT), Fibrinogen (FIB) and Thrombin Time (TT). The above indicators were collected by venous blood sampling at about 8:00 a.m. after fasting for $10 \mathrm{~h}$ and the coagulation indicators were detected by
Sysmex-2100 automatic coagulometer. The final data were collected by 2 postgraduate students by a two person dual machine built Excel sheet and proofread by the software.

\section{Statistical processing:}

The data were analyzed by Statistical Package for the Social Sciences (SPSS) 26.0. Measurement data were tested for normality by the D'Agostino test and normally distributed indexes were expressed as Mean \pm Standard Deviation (SD) and comparisons between groups were made by the independent samples t-test and comparisons among multiple groups were made by univariate Analysis Of Variance (ANOVA). The count data were expressed as percentage (\%) and the comparison between groups was performed using chi square test. $\mathrm{p}<0.05$ was considered statistically significant.

\section{RESULTS AND DISCUSSION}

Comparison of each index between groups was observed. Between the two groups, age, PT, APTT, TT and FIB followed a normal distribution by normal test and after t-test, we found that the experimental group had significantly lower age $(\mathrm{p}<0.05)$, PT and APTT levels $(p<0.05)$ and FIB levels $(p<0.05)$ than the control group, while there was no significant difference in TT levels between the two groups ( $p>0.05)$. The difference in gender and history of previous hypertension between the two groups was statistically significant $(p<0.05)$, where the percentage of males in the experimental group was significantly higher and the prevalence of history of hypertension was significantly lower than that of the control group, while there was no significant difference in the distribution of whether the two groups were smoking, drinking or not and had history of diabetes mellitus ( $>0.05$ ) (Table 1).

Univariate analysis identified PT, APTT and FIB as risk factors for the development of ONFH (Odds Ratio $(\mathrm{OR}) \geq 1, \mathrm{p}<0.05$ ). Further dividing the PT, APTT, TT and FIB levels into four classes, binary logistic regression analysis was performed and found that the risk of developing ONFH in the TT16. 10-17.00 group and 17.00-17.80 group was 1.907 times of $<16.1$ group $(\mathrm{OR}=1.907,95 \%$ Confidence Interval (CI): 1.0872.014, $\mathrm{p}<0.001), 1.541$ times $(\mathrm{OR}=1.541,95 \% \mathrm{CI}$ : $1.062-1.965, \mathrm{p}=0.016$ ); the risk of developing ONFH in the $\mathrm{FIB} \geq 4.15$ group, 3.50-4.15 group was 3.293 times of $<2.96$ group $(\mathrm{OR}=3.293,95 \% \mathrm{CI}: 2.782-$ 5.917, $\mathrm{p}<0.001), 2.092$ times $(\mathrm{OR}=2.092,95 \% \mathrm{CI}$ : $1.528-2.191, \mathrm{p}<0.001)$; the risk of developing $\mathrm{ONFH}$ 
in the PT 12.50-13.00 group and 13.00-13.70 group was 1.772 times of $<12.5$ group $(\mathrm{OR}=1.772,95 \% \mathrm{CI}$ : 1.079-2.079, $\mathrm{p}<0.001), 1.648$ times $(\mathrm{OR}=1.648,95 \%$ CI: 1.005-1.894, $\mathrm{p}=0.034)$; the risk of APTT developing ONFH in the 34.00-37.10 group and 37.10-40.60 group was 1.808 times of $<34.00$ group $(\mathrm{OR}=1.808,95 \% \mathrm{CI}$ : $1.341-2.012, \mathrm{p}<0.001), 1.483$ times $(\mathrm{OR}=1.483,95 \%$ CI: 1.104-1.569, $\mathrm{p}=0.008$ ), that is the lower the PT and APTT level, the higher the FIB level, the more likely it was to develop ONFH $(\mathrm{OR}>1, \mathrm{p}<0.05)$ and the effect of TT on ONFH occurrence was not significant $(\mathrm{p}>0.05)$ (Table 2).

Multivariate analysis of coagulation function and the occurrence of ONFH after age correction were compared. After age adjustment, PT, APTT, TT and FIB were found to be independent influences on the occurrence of $\mathrm{ONFH}$ as PT $(\mathrm{OR}=1.124,95 \% \mathrm{CI}$ : $1.027,1.146, \mathrm{p}=0.007)$, APTT $(\mathrm{OR}=1.432,95 \%$ CI: $1.135,1.928, p=0.039)$, TT $(\mathrm{OR}=1.016,95 \%$ CI: $1.006,1.079, \mathrm{p}=0.041)$, FIB (OR=1.392, $95 \%$ CI: $1.128,1.719, \mathrm{p}=0.002)$. For male, PT, APTT, TT and FIB were independent influencing factors for the occurrence of $\mathrm{ONFH}$, as $\mathrm{PT}(\mathrm{OR}=1.176,95 \% \mathrm{CI}$ : $1.085,1.391, \mathrm{p}=0.003)$, APTT $(\mathrm{OR}=1.216,95 \% \mathrm{CI}$ : $1.045,1.992, \mathrm{p}=0.031)$, TT $(\mathrm{OR}=1.035,95 \% \mathrm{CI}$ : $1.002,1.086, \mathrm{p}=0.011)$, FIB $(\mathrm{OR}=1.351,95 \% \mathrm{CI}$ : 0.945, 1.933, $\mathrm{p}=0.009$ ). For female, PT, APTT and FIB were independent influencing factors for the occurrence of $\mathrm{ONFH}$, as PT (OR=1.107, $95 \% \mathrm{CI}: 1.015,1.165$, $\mathrm{p}=0.024)$, APTT $(\mathrm{OR}=1.015,95 \% \mathrm{CI}: 1.003,1.029$, $\mathrm{p}=0.002)$, TT $(\mathrm{OR}=1.011,95 \%$ CI: 0.875, 1.168, $\mathrm{p}=0.882)$, FIB $(\mathrm{OR}=1.403,95 \%$ CI: 1.078, 1.825, $\mathrm{p}=0.012)$ (Table 3$)$.

TABLE 1: THE BASIC CLINICAL CHARACTERISTICS BETWEEN THE TWO GROUPS

\begin{tabular}{|c|c|c|c|c|c|}
\hline & Total & Control group & $\begin{array}{l}\text { Experimental } \\
\text { group }\end{array}$ & $\begin{array}{c}\mathrm{U} \text { value/T value } / \chi^{2} \\
\text { value }\end{array}$ & $p$ value \\
\hline Number of cases & 467 & 243 & 224 & & \\
\hline Age & $70.56 \pm 11.35$ & $75.39 \pm 9.63$ & $65.31 \pm 10.76$ & -10.679 & $<0.001$ \\
\hline Gender & 467 & 243 & 224 & 12.714 & $<0.001$ \\
\hline Male & 158 (33.8 \%) & $64(26.3 \%)$ & 94 (42.0\%) & & \\
\hline Female & 309 (66.2 \%) & 179 (73.7 \%) & 130 (58.0 \%) & & \\
\hline PT & $13.06 \pm 1.28$ & $13.19 \pm 1.37$ high & $12.92 \pm 1.18$ & -2.277 & $\begin{array}{c}0.023 \\
(0.01<p<0.05)\end{array}$ \\
\hline APTT & $37.33 \pm 5.46$ & $37.88 \pm 6.29$ high & $36.74 \pm 4.33$ & -2.295 & $\begin{array}{c}0.022 \\
(0.01<p<0.05)\end{array}$ \\
\hline TT & $17.10 \pm 1.84$ & $17.24 \pm 1.76$ & $16.95 \pm 1.91$ & -1.739 & $0.083(>0.05)$ \\
\hline FIB & $3.57 \pm 1.00$ & $3.47 \pm 1.06$ low & $3.97 \pm 0.92$ & 2.165 & $0.003(<0.01)$ \\
\hline Hypertension & 467 & 243 & 224 & 9.841 & $0.002(<0.01)$ \\
\hline Yes & 225 (48.2 \%) & 134 (55.1 \%) high & 91 (40.6\%) & & \\
\hline None & 242 (51.8 \%) & 109 (44.9 \%) & 133 (59.4 \%) & & \\
\hline Diabetes mellitus & 467 & 243 & 224 & 0.201 & $0.654(>0.05)$ \\
\hline Yes & 47 (10.1 \%) & 23 (9.5\%) low & 24 (10.7 \%) & & \\
\hline None & 420 (89.9 \%) & 220 (90.5 \%) & 200 (89.3 \%) & & \\
\hline $\begin{array}{l}\text { History of alcohol } \\
\text { consumption }\end{array}$ & 467 & 243 & 224 & 0.179 & $0.672(>0.05)$ \\
\hline Yes & $15(3.2 \%)$ & 7 (2.9 \%) low & $8(3.6 \%)$ & & \\
\hline None & 452 (96.8 \%) & 236 (97.1 \%) & 216 (96.4 \%) & & \\
\hline Smoking history & 467 & 243 & 224 & 0.381 & $0.537(>0.05)$ \\
\hline Yes & $26(5.6 \%)$ & 12 (4.9\%) low & $14(6.3 \%)$ & & \\
\hline None & 441 (94.4 \%) & 231 (95.1 \%) & 210 (93.8 \%) & & \\
\hline
\end{tabular}


TABLE 2: BINARY LOGISTIC REGRESSION ANALYSIS BETWEEN THE LEVEL OF COAGULATION FUNCTION (CONTINUOUS VARIABLE) AND THE OCCURRENCE OF OFNH

\begin{tabular}{|c|c|c|c|c|c|c|}
\hline \multirow{2}{*}{ Age } & \multicolumn{2}{|c|}{ Total } & \multicolumn{2}{|l|}{ Male } & \multicolumn{2}{|l|}{ Female } \\
\hline & OR $(95 \% \mathrm{Cl})$ & $p$ value & OR (95 \% Cl) & $p$ value & OR $(95 \% \mathrm{Cl})$ & $\mathrm{p}$ value \\
\hline $\mathrm{TT}$ & $0.914(0.824-1.013)$ & 0.087 & & & & \\
\hline$<16.1$ & 1.0 & & 1.0 & & 1.0 & \\
\hline $16.10-17.00$ & $1.907(1.087-2.014)$ & $<0.001$ & $1.874(1.135-2.003)$ & $<0.001$ & $1.912(1.372-2.104)$ & 0.004 \\
\hline $17.00-17.80$ & 1.541 (1.062-1.965) & 0.016 & $1.472(1.037-1.736)$ & 0.041 & $1.465(1.129-1.694)$ & 0.032 \\
\hline$\geq 17.80$ & $0.966(0.866-1.079)$ & 0.142 & $0.915(0.772-1.086)$ & 0.311 & $1.011(0.875-1.168)$ & 0.882 \\
\hline FIB & $1.221(1.017-1.465)$ & 0.033 & & & & \\
\hline$<2.96$ & 1.0 & & 1.0 & & 1.0 & \\
\hline $2.96-3.50$ & $1.248(0.863-1.815)$ & 0.382 & $1.153(0.549-1.924)$ & 0.413 & $1.026(0.834-1.528)$ & 0.603 \\
\hline $3.50-4.15$ & $2.092(1.528-2.191)$ & $<0.001$ & $2.051(1.369-2.293)$ & 0.007 & $1.314(0.906-2.036)$ & 0.129 \\
\hline$\geq 4.15$ & $3.293(2.782-5.917)$ & $<0.001$ & $3.513(1.945-6.304)$ & $<0.001$ & 3.027 (1.708-6.359) & $<0.001$ \\
\hline PT & $1.039(1.019-1.179)$ & 0.026 & & & & \\
\hline$<12.50$ & 1.0 & & 1.0 & & 1.0 & \\
\hline $12.50-13.00$ & $1.772(1.079-2.079)$ & $<0.001$ & $1.764(1.029-1.905)$ & $<0.001$ & $1.912(1.487-2.039)$ & $<0.001$ \\
\hline $13.00-13.70$ & $1.648(1.005-1.894)$ & 0.034 & $1.464(1.008-1.659)$ & 0.045 & $1.382(1.005-1.520)$ & $<0.001$ \\
\hline$\geq 13.70$ & $0.974(0.827-1.146)$ & 0.747 & $0.976(0.685-1.391)$ & 0.893 & $0.967(0.803-1.165)$ & 0.724 \\
\hline APTT & $1.262(1.130-1.995)$ & 0.025 & & & & \\
\hline$<34.00$ & 1.0 & & 1.0 & & 1.0 & \\
\hline $34.00-37.10$ & $1.808(1.341-2.012)$ & $<0.001$ & $1.910(1.277-1.945)$ & $<0.001$ & $1.912(1.462-2.205)$ & 0.014 \\
\hline $37.10-40.60$ & $1.483(1.104-1.569)$ & 0.008 & $1.170(1.003-1.239)$ & 0.003 & $1.574(1.104-1.711)$ & 0.023 \\
\hline$\geq 40.60$ & $0.972(0.935-1.009)$ & 0.139 & $0.916(0.845-0.992)$ & 0.031 & $0.985(0.943-1.029)$ & 0.502 \\
\hline
\end{tabular}

TABLE 3: MULTIVARIATE ANALYSIS OF COAGULATION FUNCTION AND THE OCCURRENCE OF ONFH AFTER AGE CORRECTION

\begin{tabular}{lcccccc}
\hline \multirow{2}{*}{ Variable } & \multicolumn{2}{c}{ Total } & \multicolumn{2}{c}{ Male } & \multicolumn{2}{c}{ Female } \\
\cline { 2 - 7 } & OR $(95 \% \mathrm{Cl})$ & p value & OR $(95 \% \mathrm{Cl})$ & p value & OR $(95 \%$ Cl) & p value \\
\hline PT & $1.124(1.027-1.146)$ & 0.007 & $1.176(1.085-1.391)$ & 0.003 & $1.107(1.015-1.165)$ & 0.024 \\
APTT & $1.432(1.135-1.928)$ & 0.039 & $1.216(1.045-1.992)$ & 0.031 & $1.015(1.003-1.029)$ & 0.002 \\
TT & $1.016(1.006-1.079)$ & 0.041 & $1.035(1.002-1.086)$ & 0.011 & $1.011(0.875-1.168)$ & 0.882 \\
FIB & $1.392(1.128-1.719)$ & 0.002 & $1.351(0.945-1.933)$ & 0.009 & $1.403(1.078-1.825)$ & 0.012 \\
\hline
\end{tabular}

Multivariate analysis between the level of coagulation function and the occurrence of ONFH after multivariate correction was shown here. After adjustment for age, smoking, drinking, hypertension and history of diabetes mellitus, the PT, APTT, TT and FIB were found to be independent predictors of the development of ONFH, with the PT $(\mathrm{OR}=1.030,95 \% \mathrm{CI}: 1.007,1.11$, $\mathrm{p}=0.0043)$, APTT $(\mathrm{OR}=1.164,95 \%$ CI: 1.092, 1.204, $\mathrm{p}=0.015,95 \%$ CI: 1.164, 1.092, 1.204, $\mathrm{p}=0.015)$, TT (OR=1.106, $95 \%$ CI: 1.021, 1.145, $\mathrm{p}=0.021)$ and FIB $(\mathrm{OR}=1.372,95 \% \mathrm{CI}: 1.104,1.704, \mathrm{p}=0.004)$. For male, PT, APTT, TT and FIB were independent influencing factors for the occurrence of $\mathrm{ONFH}$, as $\mathrm{PT}(\mathrm{OR}=1.383$, $95 \%$ CI: 1.024, 1.622, $\mathrm{p}=0.013)$, APTT $(\mathrm{OR}=1.216$, $95 \%$ CI: 1.140, 1.998, $\mathrm{p}=0.046)$, TT $(\mathrm{OR}=1.013,95$ \% CI: 1.007, 1.092, $\mathrm{p}=0.023)$, FIB (OR=1.300, 95 $\%$ CI: $0.894,1.890, \mathrm{p}=0.016)$. For female, PT, APTT and FIB were independent influencing factors for the occurrence of ONFH, PT (OR=1.021, $95 \%$ CI: 1.006, $1.098 \mathrm{p}=0.025)$. APTT $(\mathrm{OR}=1.106,95 \% \mathrm{CI}: 1.032$, 1.122, $\mathrm{p}=0.029)$, TT $(\mathrm{OR}=0.913,95 \% \mathrm{CI}: 0.771$, 1.081, $\mathrm{p}=0.292)$, FIB (OR=1.318, $95 \%$ CI: 1.003, 1.731, $\mathrm{p}=0.047)$ (Table 4). 
After adjusting for gender, age, whether they smoked, whether they drank alcohol, whether they had a history of hypertension and diabetes mellitus, PT, APTT and FIB were found to be independent risk factors for the development of ONFH and for different gender, PT, APTT and FIB levels remained independent risk factors for the development of ONFH (Table 5).
Predictive value of PT, APTT and FIB for the occurrence of ONFH was compared. The area under the curve (AUC) for PT, APTT, FIB, PT+APTT, $\mathrm{PT}+\mathrm{FIB}$, APTT+FIB， PT+APTT+FIB were 0.555 , $0.577,0.602,0.609,0.646,0.656,0.660$, respectively, all were statistically significant $(\mathrm{p}<0.05)$, where PT + APTT + FIB has the highest AUC, which is better than other combinations of detection (Table 6).

TABLE 4: MULTIVARIATE ANALYSIS BETWEEN THE LEVEL OF COAGULATION FUNCTION AND THE OCCURRENCE OF ONFH AFTER MULTIVARIATE CORRECTION

\begin{tabular}{lcccccc}
\hline \multirow{2}{*}{ Variable } & \multicolumn{2}{c}{ Total } & \multicolumn{2}{c}{ Male } & \multicolumn{2}{c}{ Female } \\
\cline { 2 - 7 } & OR $(95 \% \mathrm{Cl})$ & $\mathrm{p}$ value & OR $(95 \% \mathrm{CI})$ & $\mathrm{p}$ value & OR $(95 \% \mathrm{CI})$ & $\mathrm{p} \mathrm{value}$ \\
\hline PT & $1.030(1.007-1.117)$ & 0.043 & $1.383(1.024-1.622)$ & 0.013 & $1.021(1.006-1.098)$ & 0.025 \\
APTT & $1.164(1.092-1.204)$ & 0.015 & $1.216(1.140-1.998)$ & 0.046 & $1.106(1.032-1.122)$ & 0.029 \\
TT & $1.106(1.021-1.145)$ & 0.021 & $1.013(1.007-1.092)$ & 0.023 & $0.913(0.771-1.081)$ & 0.292 \\
FIB & $1.372(1.104-1.704)$ & 0.004 & $1.300(0.894-1.890)$ & 0.016 & $1.318(1.003-1.731)$ & 0.047 \\
\hline
\end{tabular}

TABLE 5: MULTIVARIATE ANALYSIS BETWEEN THE LEVEL OF COAGULATION FACTORS AND THE OCCURRENCE OF ONFH AFTER MULTIVARIATE CORRECTION

\begin{tabular}{lcc}
\hline \multirow{2}{*}{ Variable } & \multicolumn{2}{c}{ Total } \\
\cline { 2 - 3 } & OR $(95 \% \mathrm{Cl})$ & $\mathrm{P}$ value \\
\hline PT & $1.023(1.004-1.117)$ & 0.030 \\
APTT & $1.118(1.030-2.908)$ & 0.043 \\
TT & $0.934(0.827-1.055)$ & 0.271 \\
FIB & $1.361(1.095-1.691)$ & 0.005 \\
\hline
\end{tabular}

TABLE 6: PREDICTIVE VALUE OF PT, APTT AND FIB FOR THE OCCURRENCE OF ONFH

\begin{tabular}{lcc}
\hline \multirow{2}{*}{ Items } & \multicolumn{2}{c}{ Total } \\
\cline { 2 - 3 } PT & AUC $(95 \% \mathrm{Cl})$ & $\mathrm{p}$ value \\
APTT & $0.555(0.501-0.609)$ & 0.040 \\
FIB & $0.577(0.525-0.629)$ & 0.004 \\
PT+APTT & $0.602(0.569-0.670)$ & $<0.001$ \\
PT+FIB & $0.609(0.557-0.662)$ & $<0.001$ \\
APTT+FIB & $0.646(0.595-0.696)$ & $<0.001$ \\
PT+APTT+FIB & $0.656(0.606-0.705)$ & $<0.001$ \\
\hline
\end{tabular}


Pathogenesis of ONFH was discussed below. An intergroup comparison of coagulation indicators in patients with ONFH was performed and results were shown here. The two indicators, PT and APTT, were lower in the experimental group than in the control group. The FIB indicator was higher than that of the control group and the difference had statistical significance $(p<0.05)$. This indicates that ONFH is characterized by significant differences in coagulation (PT, APTT, FIB) indicators, which coincides with a previous study reported by Huitian et al. ${ }^{[11]}$. Several current studies have demonstrated abnormalities in coagulation in hormonal ONFH and alcoholic ONFH ${ }^{[12,13]}$. As to the role played by coagulation function in it, it is currently inconclusive. But even in the absence of hormonal and alcohol effects, there are studies indicating that there is still a correlation between coagulation abnormalities and the presence of $\mathrm{ONFH}^{[14]}$. Common coagulation functions include APTT, PT, FIB and TT indicators.

PT refers to the addition of excess tissue thromboplastin and calcium ions to platelet poor plasma and the conversion of prothrombin to thrombin, resulting in the time required for plasma coagulation. PT assay is a sieving test to check the function of the body's extrinsic coagulation system with and without disorders, while APTT refers to the observation of the time required for plasma coagulation by adding APTT reagent to the examined plasma. APTT is the most sensitive and commonly used screening test for measuring the endogenous coagulation system. PT, APTT and FIB in this study were all found to be risk factors for ONFH after adjusted and unadjusted multivariate analysis. Whether male or female patients with PT, APTT and FIB remain risk factors for $\mathrm{ONFH}$, regarding the pathogenic mechanism of ONFH caused by abnormalities in the indexes of PT, APTT and FIB, it is believed that the following aspects can be considered: Abnormalities in the coagulation indexes of PT and APTT can indirectly respond to abnormalities in the coagulation factors. Some studies have demonstrated that ghrelin levels were significantly lower and von Willebrand factor (vWF), Plasminogen Activator Inhibitor 1 (PAI-1) and C-reactive protein (CRP) levels were significantly higher in patients with non-traumatic ONFH. Plasma levels of ghrelin, vWF and PAI-1 were changed in patients with non-traumatic ONFH. Ghrelin combined with $\mathrm{VWF}$ may be involved in the pathogenesis of nontraumatic $\mathrm{ONFH}^{[15]}$. vWF is a von Willebrand factor and its role in addition to mediating platelet activated aggregation, can also bind to antihemophilic factor (FVIII), which protects it from degradation and factor FVIII is a major factor involved in the endogenous coagulation process, the increase of which can lead to a relative shortening of APTT, an increased probability of forming thrombus and an increased risk of ONFH. Yet another study showed that thrombotic Factor V Leiden (FVL) mutations are commonly associated with hip osteonecrosis ${ }^{[16]}$ and that the coagulation factor $\mathrm{V}$, a protein factor in the coagulation process that sits at the intersection of the extrinsic and intrinsic coagulation pathways, is able to accelerate and promote prothrombin activation and thrombin generation. The mutant gene, FVL, is formed when the Factor V gene is mutated. FVL combats the active effects of thrombin Activated Protein C (APC), which leads to abnormal blood coagulation, thrombophilia and hypercoagulability, and finally to $\mathrm{ONFH}^{[17,18]}$. Studies have compared the degree of reduction in thrombosis and fibrinolysis between patients with idiopathic and secondary osteonecrosis and showed that inherited thrombotic factor VIII and low fibrinolytic Lipoprotein (a) (LP (a)) were more common in patients with idiopathic osteonecrosis. High factor VIII, FVL heterozygosity and resistance to APC (all inherited thrombophilias) are more common in patients with secondary osteonecrosis. Inherited thrombophilia and decreased fibrinolysis are risk factors for idiopathic and secondary $\mathrm{ONFH}^{[19]}$. FIB also known as coagulation factor $\mathrm{I}$, is a major protein in the coagulation process, which is the most abundant coagulation factor in plasma, the main physiological function is to directly participate in the coagulation process in vivo as a coagulation factor. Starklint et al. ${ }^{[20]}$ found fibrin thrombi in areas of osteonecrosis and FIB was enzymatically converted to fibrin and then fibrin thrombi by thrombin, thus also indirectly reflecting the role of coagulation abnormalities in ONFH.

In summary, ONFH is closely related to coagulation function and coagulation disorders may participate in the mechanism of its occurrence and development, which should be paid more attention in the clinic. However, because the sample size of this study was relatively small, the conclusions need to be further investigated.

\section{Conflicts of interest:}

The authors declared no conflict of interest. 


\section{REFERENCES}

1. Leo M, Milena F, Ruggero C, Stefania S, Giancarlo T. Biophysical stimulation in osteonecrosis of the femoral head. Indian J Orthop 2009;43(1):17-21.

2. Zhao D, Zhang F, Wang B, Liu B, Li L, Kim SY, et al. Guidelines for clinical diagnosis and treatment of osteonecrosis of the femoral head in adults (2019 version). J Orthop Translat 2020;21:100-10.

3. Cohen-Rosenblum A, Cui Q. Osteonecrosis of the Femoral Head. Orthop Clin North Am 2019;50(2):139-49.

4. Zalavras CG, Lieberman JR. Osteonecrosis of the femoral head: evaluation and treatment. J Am Acad Orthop Surg 2014;22(7):455-64.

5. Xu J, Gong H, Lu S, Deasey MJ, Cui Q. Animal models of steroid-induced osteonecrosis of the femoral head-a comprehensive research review up to 2018. Int Orthop 2018;42(7):1729-37.

6. Krenn V, Müller S, Krenn VT, Hempfling H. Pathophysiology of aseptic femoral head necrosis: Pathogenesis and histopathological differential diagnosis. Orthopade 2018;47(9):710-6.

7. Severyns M, Gayet LE. Aseptic osteonecrosis of the femoral had in patients with sickle cell anemia. Morphologie 2021;105(349):94-101.

8. Jiang J. Correlation analysis between the levels of macrophages and neutrophils in peripheral blood and ONFH. Nanchang Univ 2020.

9. Zhang Q, Jin LV, Jin L. Role of coagulopathy in glucocorticoidinduced osteonecrosis of the femoral head. J Int Med Res 2018;46(6):2141-8.

10. Meloni MC, Hoedemaeker WR, Fornasier V. Failed vascularized fibular graft in treatment of osteonecrosis of the femoral head. A histopathological analysis. Joints 2016;4(1):24-30.

11. Huitian L. Coagulation tests and clinical significance in patients with ONFH. J Chin Med 2014;29(B12):431-2.
12. Chikvatia L, Avazashvili N, Obgaidze G. Steroid induced osteonecrosis of the femoral head. Mol Med Rep 2012;6(3):6114.

13. Huisong L. Pathogenesis of alcoholic ONFH and related studies. Soochow Univ 2003.

14. Garcia FL, Ramalli EL, Picado CHF. Coagulation disorders in patients with femoral head osteonecrosis. Acta Ortop Bras 2013;21(1):43-5.

15. Li C, Shen L, Yang Y, Shuai B, Xu X, Ma C. Plasma ghrelin and von Willebrand factor levels in patients with non-traumatic osteonecrosis of the femoral head. Hip Int 2015;25(1):76-81.

16. Glueck CJ, Freiberg RA, Boriel G, Khan Z, Brar A, Padda J, et al. The role of the factor $\mathrm{V}$ Leiden mutation in osteonecrosis of the hip. Clin Appl Thromb Hemost 2013;19(5):499-503.

17. Reitsma PH, van de Stolpe A. From gene to disease; risk factors for venous thrombosis: factor $\mathrm{V}$ Leiden and prothrombin 20210A. Ned Tijdschr Geneeskd 2001;145(38):1843-5.

18. Jody Lynn Kujovich. Factor V Leiden thrombophilia. Genet Med 2011;13(1):1-16.

19. Glueck CJ, Freiberg RA, Wang P. Heritable thrombophiliahypofibrinolysis and osteonecrosis of the femoral head. Clin Orthop Relat Res 2008;466(5):1034-40.

20. Starklint H, LaustenGS, Arnoldi CC. Microvascular obstruction in avascular necrosis. Immunohistochemistry of 14 femoral heads. Acta Orthop Scand 1995;66(1):9-12.

This is an open access article distributed under the terms of the Creative Commons Attribution-NonCommercial-ShareAlike 3.0 License, which allows others to remix, tweak, and build upon the work non-commercially, as long as the author is credited and the new creations are licensed under the identical terms

This article was originally published in a special issue, "Novel Therapeutic Approaches in Biomedicine and Pharmaceutical Sciences" Indian J Pharm Sci 2021:83(6) Spl Issue "54-60" 